\title{
Application of symmetric analytic functions to spectra of linear operators
}

\begin{abstract}
Burtnyak I. ${ }^{1}$, Chernega I. ${ }^{2, \bigotimes}$, Hladkyi V. ${ }^{1}$, Labachuk O. ${ }^{1}$, Novosad Z. ${ }^{3}$
The paper is devoted to extension of the theory of symmetric analytic functions on Banach sequence spaces to the spaces of nuclear and $p$-nuclear operators on the Hilbert space. We introduced algebras of symmetric polynomials and analytic functions on spaces of $p$-nuclear operators, described algebraic bases of such algebras and found some connection with the Fredholm determinant of a nuclear operator. In addition, we considered cases of compact and bounded normal operators on the Hilbert space and discussed structures of symmetric polynomials on corresponding spaces.

Key words and phrases: symmetric analytic function on a Banach space, $p$-nuclear operator, Fredholm determinant.
\end{abstract}

\footnotetext{
${ }^{1}$ Vasyl Stefanyk Precarpathian National University, 57 Shevchenka str., 76018, Ivano-Frankivsk, Ukraine

2 Pidstryhach Institute for Applied Problems of Mechanics and Mathematics, 3b Naukova str., 79060, Lviv, Ukraine

${ }^{3}$ Lviv University of Trade and Economics, 10 Tuhan-Baranovsky str., 79005, Lviv, Ukraine

$\checkmark$ Corresponding author

E-mail: ivan.burtnyakepnu.edu.ua (Burtnyak I.), icherneha@ukr.net (Chernega I.),

kartesiusr@gmail. com (Hladkyi V.), olabachuk@gmail . com (Labachuk O.),

zoryana.math@gmail. com (Novosad Z.)
}

\section{Introduction and preliminaries}

Investigations of symmetric analytic function of infinitely many variables is important regarding to possible applications in Data Science, Statistical Mechanics, Neural-Networks and other branches of knowledge [18,29]. By this reason, symmetric polynomials and analytic functions give us useful tools. On the other hand, the amount and behavior of symmetric analytic functions on a Banach space with a symmetric structure essentially depend on the particular space [21].

Symmetric polynomials in classic algebra means polynomials on $\mathbb{C}^{n}$ which are invariant with respect to the group of permutations of basis vectors. There are two natural generalizations of the group of permutations of basis vectors for the case of Banach spaces with some symmetric structures. We consider here the cases when $X=\ell_{p}, 1 \leq p<\infty$, or $L_{\infty}[0 ; 1]$, or $c_{0}$. For the cases of sequence spaces like $\ell_{p}$ or $c_{0}$ it is naturally to consider the group $\mathcal{S}^{\infty}$ of all permutations (bijections) of $\mathbb{N}$. Every element $s \in \mathcal{S}^{\infty}$ acts on a sequence space $X$ by $s(x)=\left(x_{s(1)}, \ldots, x_{s(n), \ldots}\right)$, where $x=\left(x_{1}, \ldots, x_{n}, \ldots\right) \in X$. In the case $X=L_{\infty}[0 ; 1]$, we use the group $\Xi$ of all measurable automorphisms of $[0 ; 1]$ that preserve the Lebesgue measure. Elements $s \in \Xi$ act on $L_{\infty}[0 ; 1]$ by $s(x)=x \circ s$, where $x=x(t) \in L_{\infty}[0 ; 1]$. Let $\mathfrak{C}$ be a group $\mathcal{S}^{\infty}$ or $\Xi$ on $X=\ell_{p}$ or $L_{\infty}[0 ; 1]$, respectively. A scalar-valued function $f$ on $X$ is said to be symmetric

$\mathrm{y} \Delta \mathrm{K} 517.98$

2020 Mathematics Subject Classification: 47A75, 46G20, 46E25, 46J20.

This research was funded by the National Research Foundation of Ukraine, 2020.02/0025, 0121U111037 
if $f(s(x))=f(x)$ for every $x \in X$ and $s \in \mathfrak{C}$.

Let us recall that a function $P$ on a Banach space $X$ is an $n$-homogeneous polynomial if there is an $n$-linear map $B$ on the $n$th Cartesian power $X^{n}$ such that $P(x)=B(x, \ldots, x)$. A continuous function $f$ on $X$ is analytic if its restriction to any finite dimensional subspace is analytic. Every analytic mapping can be represented by its Taylor series expansion

$$
f(x)=\sum_{n=0}^{\infty} f_{n}(x)
$$

where $f_{n}$ are $n$-homogeneous polynomials. If an analytic function on $X$ is bounded on all bounded subsets, then it is called a function of bounded type, otherwise it is a function of unbounded type. For details on polynomials and analytic functions on Banach spaces we refer the reader to [22].

Symmetric analytic functions on Banach spaces for various symmetry groups, algebras of symmetric analytic functions and their spectra were considered by many authors [3-6,8-10,12, $14-17,19,23,25,26]$.

It is known $[17,23]$ that polynomials

$$
F_{k}(x)=\sum_{n=1}^{\infty} x_{n}^{k}, \quad k=\lceil p\rceil,\lceil p\rceil+1, \ldots,
$$

form an algebraic basis in the algebra of all symmetric continuous polynomials on $\ell_{p}$, $1 \leq p<\infty$, where $\lceil p\rceil$ is the smallest integer not less than $p$. In other words, every symmetric continuous polynomial on $\ell_{p}$ can be uniquely represented as an algebraic combination of polynomials $F_{k}, k \geq\lceil p\rceil$. On the other hand, only constants are symmetric continuous polynomials on $c_{0}$. In [17] it is proved that a continuous function on $\ell_{p}, 1 \leq p<\infty$, is symmetric if and only if it is $S_{0}^{\infty}$-symmetric, where $S_{0}^{\infty} \subset \mathcal{S}^{\infty}$ is the subgroup of finite permutations. Note that in $\ell_{\infty}$ it is not so [14]. Also, in [17] it is proved that polynomials

$$
R_{n}(x)=\int_{[0 ; 1]}(x(t))^{n} d t, \quad x \in L_{\infty}[0 ; 1], \quad n \in \mathbb{N},
$$

form an algebraic basis in the algebra of all symmetric continuous polynomials on $L_{\infty}[0 ; 1]$. The algebra of all symmetric continuous polynomials on $X$ is denoted by $\mathcal{P}_{S}(X)$. The classical combinatorial approach to symmetric polynomials can be found in [20].

Let $X=\ell_{p}, 1 \leq p<\infty$, or $c_{0}$. We can consider every element $x=\left(x_{1}, \ldots, x_{n}, \ldots\right) \in X$ as an operator $A_{x}$ of coordinate-wise multiplication on $x$ in the Hilbert space $\ell_{2}$. In other words,

$$
A_{x}(h)=\sum_{n=1}^{\infty} x_{n} h_{n} e_{n}, \quad h \in \ell_{2}
$$

where $\left(e_{n}\right)$ is the standard orthonormal basis in $\ell_{2}$. Clearly, $A_{x}$ is bounded and normal. If $X=\ell_{p}, 1 \leq p<\infty, A_{x}$ is $p$-nuclear. If $x=c_{0}$, then $A_{x}$ is a compact operator. Conversely, from the spectral theorem for compact operators [24, p. 64], we have that every normal $p$-nuclear or compact operator can be represented by (1) in an orthonormal basis $\left(e_{n}\right)$ for some $x$ in $\ell_{p}$ or $c_{0}$, respectively. 
If $X=L_{\infty}[0 ; 1]$, for every $x(t) \in L_{\infty}[0 ; 1]$, operator $A_{x}$ on $L_{2}[0 ; 1]$, defined by

$$
A_{x}(h)=x(t) h(t), \quad h(t) \in L_{2}[0 ; 1],
$$

is normal and bounded. Also, from the spectral theorem for normal operators [13, p. 444], we know that for any normal and bounded operator $A$ on a Hilbert space $H$ there exists a topological space $\Omega$, a measure $\mu$ and an isometry $U: L_{2}(\Omega, \mu) \rightarrow H$ such that $U^{-1} A U$ is a multiplication operator on $L_{2}(\Omega, \mu)$.

The main idea of this paper is to study symmetric polynomials and analytic functions on normal operators on Hilbert spaces using the representation $A_{x}, x \in X$. Such approach allows us to get an interplay between symmetric analytic functions and the Operator Theory, having some benefits for both of them. Basically, we consider the case of normal nuclear and $p$-nuclear operators. In the case of nuclear operators, symmetric polynomials can be represented by the trace of a given operator and so the definition of symmetric polynomials can be extended to the space of all nuclear operators.

Since we proceed with symmetric polynomials $\mathcal{P}_{S}(X)$, it is natural to introduce the relation of equivalence on $X$. We say that $x \sim y$ if and only if $P(x)=P(y)$ for every $P \in \mathcal{P}_{s}(X)$ (see, e.g. [8]). We denote by $\mathcal{M}_{X}$ the quotient set $X / \sim$. Hence, the equivalence on $X$ induces an equivalence on corresponding spaces of normal operators by $A_{x} \sim A_{y}$ if and only if $x \sim y$. We denote by $[x]$ the class of equivalence, containing $x$, and by $[A]$ the class of equivalence, containing $A$.

Let $A$ be a compact operator on $\ell_{2}$. Then $\sigma(A) \backslash\{0\}$ is a point spectrum of $A$ and each element of it has a finite multiplicity. Let us denote by $\mathfrak{S}(A)$ the multiset of all elements of $\sigma(A) \backslash\{0\}$ accounted with their multiplicities. That is, if $\sigma(A) \backslash\{0\}=\left\{\lambda_{1}, \ldots, \lambda_{n}, \ldots\right\}$, then

$$
\mathfrak{S}(A)=\{\underbrace{\lambda_{1}, \ldots, \lambda_{1}}_{k_{1}}, \ldots, \underbrace{\lambda_{n}, \ldots, \lambda_{n}}_{k_{n}}, \ldots\},
$$

where $k_{n}$ is the multiplicity of $\lambda_{n}$. If $x \in c_{0}$ is such that $A=A_{x}$ in some orthonormal basis of $\ell_{2}$, then we can identify the multiset $\mathfrak{S}(A)$ and the class of equivalence $[x]$.

\section{Main results}

\subsection{Nuclear and $p$-nuclear operators}

If $A$ is a normal nuclear operator on $\ell_{2}$, then the multiset $\mathfrak{S}(A)$ is absolutely summable and

$$
\operatorname{tr} A=\sum_{\lambda_{n} \in \mathfrak{S}(A)} \lambda_{n}
$$

is the trace of $A$. Let $x \in \ell_{1}$ be such that $A=A_{x}$, then

$$
\operatorname{tr} A=\operatorname{tr} A_{x}=\sum_{n=1}^{\infty} x_{n}=F_{1}(x) .
$$

Moreover, in this case, $F_{k}(x)=\operatorname{tr} A^{k}$ for every $k \in \mathbb{N}$. The similar situation is in the case of $p$-nuclear operators for $k \geq\lceil p\rceil$.

Let us denote by $N_{p}\left(\ell_{2}\right)$ the linear space of all $p$-nuclear operators and by $\mathfrak{N} N_{p}\left(\ell_{2}\right)$ the subspace of all normal $p$-nuclear operators on $\ell_{2}$.

$N_{p}\left(\ell_{2}\right)$ is a Banach space with respect to the nuclear norm $\|A\|_{p}:=\left(\operatorname{tr}\left|A^{p}\right|\right)^{1 / p}$, where $|A|=\sqrt{A A^{*}}$, and $\mathfrak{N} N_{p}\left(\ell_{2}\right)$ is a closed subspace of $N_{p}\left(\ell_{2}\right)$. We call a function $f: N_{p}\left(\ell_{2}\right) \rightarrow \mathbb{C}$ symmetric if $f(A)=f(B)$ for every $A, B$ such that $\mathfrak{S}(A)=\mathfrak{S}(B)$. 
Proposition 1. If $f$ is a symmetric function on $N_{p}\left(\ell_{2}\right)$, then for every unitary operator $U$ on $\ell_{2}$, $f\left(U A U^{-1}\right)=f(A)$.

Proof. The proof immediately follows from the fact that $\mathfrak{S}(A)=\mathfrak{S}\left(U A U^{-1}\right)$.

Let $P \in \mathcal{P}\left(\ell_{p}\right)$. Denote by $\widetilde{P}=\iota(P)$ a polynomial on $\mathfrak{N} N_{p}\left(\ell_{2}\right)$ such that $P(x)=\widetilde{P}\left(A_{x}\right)$ for every $x \in \ell_{p}$. We denote by $\mathcal{P}_{S}\left(\mathfrak{N} N_{p}\left(\ell_{2}\right)\right)$ the algebra of all symmetric polynomials on $\mathfrak{N N} N_{p}\left(\ell_{2}\right)$.

Theorem 1. The mapping $\iota$ is an algebra isomorphism from $\mathcal{P}_{s}\left(\ell_{p}\right)$ to $\mathcal{P}_{s}\left(\mathfrak{N} N_{p}\left(\ell_{2}\right)\right)$. In particular, polynomials $\widetilde{F}_{k}(A)=\operatorname{tr} A^{k}$ form an algebraic basis in the algebra of all continuous symmetric polynomials on $\mathfrak{N} N_{p}\left(\ell_{2}\right)$.

Proof. Let $P \in \mathcal{P}_{s}\left(\ell_{p}\right)$, and $\operatorname{deg} P=m$. Then $P$ is an algebraic combination of polynomials $\left\{F_{k}\right\}$, that is, there is a polynomial $q$ of $m-\lceil p\rceil+1$ variables such that

$$
P(x)=q\left(F_{\lceil p\rceil}(x), F_{\lceil p\rceil+1}(x), \ldots, F_{m}(x)\right) .
$$

Then

$$
\widetilde{P}(A)=q\left(\operatorname{tr} A^{\lceil p\rceil}, \operatorname{tr} A^{\lceil p\rceil+1}, \ldots, \operatorname{tr} A^{m}\right) .
$$

Hence, $\widetilde{P}$ is a symmetric polynomial on $\mathfrak{N} N_{p}\left(\ell_{2}\right)$, and if $P \neq 0$, then $\widetilde{P} \neq 0$, and so, $\iota$ is injective. If $Q \in \mathcal{P}_{s}\left(\mathfrak{N} N_{p}\left(\ell_{2}\right)\right)$, then we can define $P(x)=Q\left(A_{x}\right)$ and so $Q=\widetilde{P}$. Thus, $P$ is surjective. In addition, it is easy to see that $\iota$ is linear and multiplicative. So it is an algebra isomorphism.

Corollary 1. The isomorphism $\iota$ can be extended to a continuous isomorphism from the algebra of all entire symmetric function $H_{s}\left(\ell_{p}\right)$ to the algebra of entire symmetric functions $H_{s}\left(\mathfrak{N N} N_{p}\left(\ell_{2}\right)\right)$ by

$$
\widetilde{f}(A)=\sum_{n=0}^{\infty} \widetilde{f}_{n}(A)
$$

where $f_{n}$ are the Taylor's polynomials of $f \in H_{s}\left(\ell_{p}\right)$. If $f$ is of bounded type, then $\tilde{f}$ is of bounded type.

Proof. Since $f_{n}(x)=\widetilde{f}_{n}\left(A_{x}\right)$ for every $x \in \ell_{p}$, the series (2) converges at $A=A_{x}$ for every $x \in \ell_{p}$. Thus $\tilde{f}$ is well-defined on $\mathfrak{N} N_{p}\left(\ell_{2}\right)$ and analytic. If $f$ is bounded on all bounded subsets of $\ell_{p}$, then the radius of boundedness at zero is equal to

$$
\varrho_{0}(f)=\left(\limsup _{n \rightarrow \infty}\left\|f_{n}\right\|^{1 / n}\right)^{-1}=\infty
$$

But $\left\|A_{x}\right\|_{p}=\|x\|_{\ell_{p}}$ and so $\left\|f_{n}\right\|=\left\|\widetilde{f}_{n}\right\|$, hence, $\varrho_{0}(\widetilde{f})=\infty$. That is, $\widetilde{f}$ is of bounded type.

Note that on every infinite dimensional Banach space always there are analytic functions of unbounded type [1,2]. Also there are symmetric analytic functions of unbounded type on $\ell_{p}, 1 \leq p<\infty,[11,27]$, but no on $L_{\infty}[0 ; 1][28]$.

From Theorem 1 we have the following criterium, if $[A]=[B]$ for some $A, B \in \mathfrak{N} N_{p}\left(\ell_{2}\right)$. 
Corollary 2. Let $A, B \in \mathfrak{N} N_{p}\left(\ell_{2}\right)$. Then $[A]=[B]$ if and only if there is some $m_{0} \in \mathbb{N}$ such that $\operatorname{tr} A^{m}=\operatorname{tr} B^{m}$ for every $m \geq m_{0}$.

Proof. Suppose that $\operatorname{tr} A^{m}=\operatorname{tr} B^{m}$ for every $m \geq m_{0}$. Let $A=A_{x}$ and $B=B_{y}$ for some $x, y \in \ell_{p}$. Then, $F_{m}(x)=F_{m}(y)$ for every $m \geq m_{0}$. By [3, Theorem 1.3] $[x]=[y]$ and so $[A]=[B]$. The inverse statement is clear.

From Corollary 2 it follows that if $\operatorname{tr} A^{m}=0$ for all $m$ which are greater than a given natural number $m_{0}$, then $A=0$. However, using ideas in [3,9] it is possible to construct a sequence $\left(A_{n}\right) \subset \mathfrak{N} N_{p}\left(\ell_{2}\right)$ such that

$$
\lim _{n \rightarrow \infty} \operatorname{tr} A_{n}^{m} \neq 0
$$

for a finite number of $m$. Indeed, let $m \geq p$ and

$$
A_{n}^{(m)}(h)=\frac{1}{\sqrt[m]{n}} \sum_{i=1}^{n} h_{i} e_{i}
$$

Then,

$$
\operatorname{tr}\left(A_{n}^{(m)}\right)^{k}=\frac{n}{n^{k / m}}=n^{1-k / m}
$$

Thus, for $k>m, \operatorname{tr}\left(A_{n}^{(m)}\right)^{k} \rightarrow 0$ as $n \rightarrow \infty$ and for $k=m$,

$$
\lim _{n \rightarrow \infty} \operatorname{tr}\left(A_{n}^{(m)}\right)^{m}=1
$$

Note, that the sequence $\left(A_{n}^{(m)}\right)$ is bounded for a fixed $m$ only if $p$ is integer and $m=p$.

Since symmetric polynomials of $A \in \mathfrak{N} N_{p}\left(\ell_{2}\right)$ can be defined by the trace of $A^{k}, k \geq p$, they formally may be applied to any operators in $N_{p}\left(\ell_{2}\right)$. Let us denote by $\widehat{P}$ the extension of a symmetric polynomial $P$ to $N_{p}\left(\ell_{2}\right)$ such that $\widehat{F}_{k}=\operatorname{tr} A^{k}, k \geq p$, and the operator $\widehat{\imath}: P \mapsto \widehat{P}$ as an algebra homomorphism. Clearly, that $\|\widehat{P}\|_{N_{p}\left(\ell_{2}\right)}=\|P\|_{\ell_{p}}$, and the restriction of $\widehat{P}$ to $\mathfrak{N} N_{p}\left(\ell_{2}\right)$ is equal to $\widetilde{P}$.

Corollary 3. Every symmetric analytic function $f$ on $\ell_{p}$ can be extended to a symmetric function $\widehat{f}$ on $N_{p}\left(\ell_{2}\right)$, and if $f$ is of bounded type, then $\widehat{f}$ is of bounded type too.

Proof. Like in the proof of Corollary 1, we can see that

$$
\widehat{f}(A)=\sum_{n=0}^{\infty} \widehat{f}_{n}(A), \quad A \in N_{p}\left(\ell_{2}\right),
$$

is the required extension.

Let us consider the case $p=1$ more detailed. In the algebra of symmetric polynomials on $\ell_{1}$ we have other algebraic bases, the basis of elementary symmetric polynomials

$$
G_{n}(x)=\sum_{i_{1}<\cdots<i_{n}} x_{i_{1}} \cdots x_{i_{n}}, \quad x \in \ell_{1},
$$

and the basis of complete symmetric polynomials

$$
H_{n}(x)=\sum_{i_{1} \leq \cdots \leq i_{n}} x_{i_{1}} \cdots x_{i_{n}}, \quad x \in \ell_{1} .
$$


The relation between $\left(F_{n}\right),\left(H_{n}\right)$ and $\left(G_{n}\right)$ can be given by the Newton formulas

$$
n G_{n}(x)=\sum_{k=1}^{n}(-1)^{k+1} F_{k} G_{n-k}, \quad n H_{n}(x)=\sum_{k=1}^{n} F_{k} H_{n-k}, \quad n \in \mathbb{N} .
$$

Thus setting $\widehat{G}_{1}=\widehat{H}_{1}=\widehat{F}_{1}$, we can define $\widehat{G}_{n}$ and $\widehat{H}_{n}$ by induction using the Newton formulas.

It is known [10] that for every fixed $x \in \ell_{1}$,

$$
\mathcal{G}(x)(t)=\sum_{n=1}^{\infty} t^{n} G_{n}(x)=\prod_{n=1}^{\infty}\left(1+x_{n} t\right), \quad t \in \mathbb{C}, \quad G_{0}=1,
$$

is an entire function of exponential type. Moreover (see, e.g. [19]),

$$
\mathcal{G}(x)(t)=\exp \left(\sum_{n=1}^{\infty}(-1)^{n+1} \frac{t^{n} F_{n}(x)}{n}\right)
$$

and

$$
\mathcal{H}(x)(t)=\sum_{n=1}^{\infty} t^{n} H_{n}(x)=\frac{1}{\mathcal{G}(-x)(t)}
$$

in the domain, where the right sides are defined.

Of course, these relations are true if we apply the isomorphism $\widehat{\imath}$. But for this case, we can rewrite it in a different form. Indeed, we know that if $\|t A\|<1$, then, in the means of functional calculus, we can write

$$
\ln (I+t A)=\sum_{n=1}^{\infty}(-1)^{n+1} \frac{t^{n} A^{n}}{n} .
$$

Thus

$$
\operatorname{tr}(\ln (I+t A))=\sum_{n=1}^{\infty}(-1)^{n+1} \frac{t^{n} \widehat{F}_{n}(x)}{n} .
$$

Hence, we have the following result.

Proposition 2. Let $A$ and $B$ are in $\mathfrak{N N} N_{1}\left(\ell_{2}\right)$. Then $[A]=[B]$ if and only if

$$
e^{\operatorname{tr}(\ln (I+t A))}=e^{\operatorname{tr}(\ln (I+t B))}
$$

for all $t \in \mathbb{C}$ such that $|t|<\min (\|A\|,\|B\|)$.

Proof. As it was shown above,

$$
e^{\operatorname{tr}(\ln (I+t A))}=\widehat{\mathcal{G}}(A)(t)=\sum_{n=1}^{\infty} t^{n} \widehat{G}_{n}(A) .
$$

So, if $e^{\operatorname{tr}(\ln (I+t A))}=e^{\operatorname{tr}(\ln (I+t B))}$ for all $t$ in some neighbourhood of zero, then $\widetilde{G}_{n}(A)=\widetilde{G}_{n}(B)$, $n \in \mathbb{N}$. Since $\left(\widetilde{G}_{n}\right)$ is an algebraic basis in $\mathcal{P}_{\mathcal{S}}\left(\mathfrak{N} N_{1}\left(\ell_{2}\right)\right)$, $[A]=[B]$.

Let us recall that the Fredholm determinant of a given nuclear operator $A$ can be defined by (see [7])

$$
\operatorname{det}(I+t A)=\prod_{\lambda_{n} \in \mathcal{S}(A)}\left(1+t \lambda_{n}\right) .
$$

Combining formulas (3)-(7) we have the following theorem. 
Theorem 2. The Fredholm determinant and its inverse of a given nuclear operator $A$ can be computed by

$$
\operatorname{det}(I+t A)=e^{\operatorname{tr}(\ln (I+t A))}=\sum_{n=1}^{\infty} t^{n} \widehat{G}_{n}(A)=\exp \left(\sum_{n=1}^{\infty}(-1)^{n+1} \frac{t^{n} \operatorname{tr} A^{n}}{n}\right)
$$

and

$$
(\operatorname{det}(I+t A))^{-1}=\widehat{\mathcal{H}}(-A)(t)=\sum_{n=1}^{\infty} t^{n} \widehat{H}_{n}(-A) .
$$

Note that formula (8) is well-known [7, p. 880]. However, using symmetric polynomials we can easily compute (8) and (9) for some nuclear operator $A$. Indeed, let $A \in N_{1}\left(\ell_{2}\right)$. First, we can calculate $\widehat{F}_{n}(A)=\operatorname{tr} A^{n}$ and using the Newton formulas, find $\widehat{G}_{n}(A)$ and $\widehat{H}_{n}(A)$, $n \in \mathbb{N}$. Next, the finite series

$$
\sum_{n=1}^{N} t^{n} \widehat{G}_{n}(A) \text { and } \sum_{n=1}^{N} t^{n}(-1)^{n} \widehat{H}_{n}(A)
$$

will approximate $\operatorname{det}(I+t A)$ and $(\operatorname{det}(I+t A))^{-1}$, respectively, as $N \rightarrow \infty$.

Let now $A$ be a self-adjoint operator in $\mathfrak{N N} N_{p}\left(\ell_{2}\right)$. Then $\sigma(A) \subset \mathbb{R}$ and if $x \in \ell_{p}$ is such that $A=A_{x}$, then all coordinates $x_{n}$ of $x$ are real. In this case, $\widetilde{G}_{n}(A)$ are real numbers for all $n \in \mathbb{N}$. The inverse statement is not true. For a given vector $x=\left(x_{1}, \ldots, x_{n}, \ldots\right) \in \ell_{p}$ we denote by $\bar{x}:=\left(\bar{x}_{1}, \ldots, \bar{x}_{n}, \ldots\right)$, where $\bar{x}_{n}$ is the complex conjugate to $x_{n}$.

Proposition 3. For a given operator $A=A_{x} \in \mathfrak{N} N_{p}\left(\ell_{2}\right)$ the values $\widetilde{G}_{n}(A)$ are real if and only if $[x]=[\bar{x}]$. Equivalently, if $\lambda \in \sigma(A)$ and $\lambda \notin \mathbb{R}$, then $\bar{\lambda} \in \sigma(A)$ having the same multiplicity.

Proof. From (3) we have that

$$
\widetilde{\mathcal{G}}\left(A_{x}\right)(t)=\mathcal{G}(x)(t)=\prod_{n=1}^{\infty}\left(1+x_{n} t\right) .
$$

Thus, if $x_{n}=\lambda \notin \mathbb{R}$ and for some $k, x_{k}=\bar{\lambda}$, then their product is real. Conversely, if all coefficients of an entire function are real, then its zeros are either real or for every non-real zero $\lambda$ the zero $\bar{\lambda}$ has the same multiplicity. But zeros of $\mathcal{G}(x)(t)$ are of the form $a_{n}=1 / x_{n}$ for $x_{n} \neq 0$.

Corollary 4. An operator $A=A_{x} \in \mathfrak{N} N_{p}\left(\ell_{2}\right)$ is self-adjoint if and only if all zeros of $\operatorname{det}(A+t I)=\widetilde{\mathcal{G}}\left(A_{x}\right)(t)$ are real.

\subsection{More general cases of operators}

If $A$ is a normal compact operator on $\ell_{2}$, then there exists an orthonormal basis in $\ell_{2}$ such that $A$ can be represented in this basis as an operator $A_{x}$ of multiplication by some vector $x \in c_{0}$. But there are no symmetric polynomials on $c_{0}$ excepting constants (see e.g. [17]). In [21] were introduced slice-symmetric polynomials and slice-symmetric $G$-analytic functions on the real $c_{0}$, which we denote by $c_{0}^{\mathbb{R}}$, by the following way. For a given $\varepsilon>0$ we denote by $\mathcal{J}_{\varepsilon}$ the following mapping from $c_{0}^{\mathbb{R}}$ to itself

$$
\mathcal{J}_{\varepsilon}\left(\sum_{n=1}^{\infty} x_{n} e_{n}\right)=\sum_{n=1}^{\infty} y_{n} e_{n}
$$


where

$$
y_{n}= \begin{cases}0, & \text { if }\left|x_{n}\right|<\varepsilon \\ x_{n}-\varepsilon, & \text { if } x_{n} \geq \varepsilon \\ x_{n}+\varepsilon, & \text { if }-x_{n} \geq \varepsilon\end{cases}
$$

In [21] it is proved that $\mathcal{J}_{\varepsilon}$ is continuous and even 1-Lipschitz. Moreover, if $\varepsilon \rightarrow 0$, then $\mathcal{J}_{\mathcal{\varepsilon}}(x) \rightarrow x$ uniformly on $c_{0}^{\mathbb{R}}$. It is easy to see that the range of $\mathcal{J}_{\mathcal{\varepsilon}}$ is in the subspace $c_{00}$ of finite sequences. Let $P$ be a symmetric polynomial on $c_{00}$ and $\varepsilon>0$. We define $P^{(\varepsilon)}(x)=$ $P\left(\mathcal{J}_{\mathcal{\varepsilon}}(x)\right)$ and call it an symmetric $\varepsilon$-slice-polynomial. In [21] it is shown that $P^{(\varepsilon)}$ is symmetric and continuous. Of course, $P^{(\varepsilon)}$ is not a polynomial.

Since symmetric polynomials $F_{n}$ are well defined on $c_{00}$ for every $n \in \mathbb{N}$ and form an algebraic basis in the algebra $\mathcal{P}\left(c_{00}\right)$ of all symmetric polynomials on $c_{00}$, we can define slice symmetric polynomials $F_{n}^{(\varepsilon)}$ on $c_{0}^{\mathbb{R}}$. Let us denote by $K S\left(\ell_{2}\right)$ the linear space of compact selfadjoint operators on $\ell_{2}$. From the spectral theorem for compact self-adjoint operators we have that if $A \in K S\left(\ell_{2}\right)$, then $A=A_{x}$ for some $x \in c_{0}^{\mathbb{R}}$. Thus, for every $\varepsilon>0$, we can define $\widetilde{P^{(\varepsilon)}}\left(A_{x}\right):=P^{(\varepsilon)}(x)$. In particular, $\widetilde{F_{n}^{(\varepsilon)}}(A)=\operatorname{tr}\left(A \circ \mathcal{J}_{\varepsilon}\right)^{n}$. The next proposition follows from definitions.

Proposition 4. For every $\varepsilon>0$, the set of symmetric $\varepsilon$-slice-polynomials is an algebra of functions and the mapping $P^{(\varepsilon)} \mapsto \widehat{P^{(\varepsilon)}}$ is an algebra homomorphism. Slice-polynomials $F_{n}^{(\varepsilon)}$ form an algebraic basis in the algebra of symmetric $\varepsilon$-slice-polynomials.

Corollary 5. Let $A, B \in K S\left(\ell_{2}\right)$. If for every $\varepsilon>0, \operatorname{tr}\left(A \circ \mathcal{J}_{\varepsilon}\right)^{n}=\operatorname{tr}\left(B \circ \mathcal{J}_{\varepsilon}\right)^{n}$, then $[A]=[B]$.

Proof. As in Corollary 2 we can prove that $\left[A \circ \mathcal{J}_{\varepsilon}\right]=\left[B \circ \mathcal{J}_{\varepsilon}\right]$ for every $\varepsilon>0$. If $[A] \neq[B]$, then there is $c>0$ such that

$$
\inf _{s \in \mathcal{S}_{0}^{\infty}}\|x-s(y)\| \geq c>0
$$

where $x$ and $y$ are such that $A=A_{x}$ and $B=B(y)$, and $s(y)=\left(y_{s(1)}, \ldots, y_{s(n)}, \ldots\right)$. Taking $\varepsilon<c$ we get a contradiction.

The general case of normal linear operators leads to the representation of any bounded normal operator as an operator of multiplication in $L_{2}[0 ; 1]$ by a function in $L_{\infty}[0 ; 1]$. In this case, the group of symmetry is the group of measurable automorphisms $\Xi$ of the interval $[0 ; 1]$ to itself which preserve the Lebesgue measure. Polynomials

$$
R_{n}(x)=\int_{[0 ; 1]}(x(t))^{n} d t, \quad x \in L_{\infty}[0 ; 1]
$$

form an algebraic basis in the algebra of all symmetric polynomials on $L_{\infty}[0 ; 1]$. However, for the complex $L_{\infty}[0 ; 1]$ the equalities $R_{n}(x)=R_{n}(y)$ for all $n \in \mathbb{N}$ does not imply that $[x]=[y]$. For example, if $x(t)=e^{2 \pi i t}$, then $R_{n}(x)=0$ for every $n$, but $x \neq 0$. For the real case, the situation is different. Indeed, if $x \in L_{\infty}^{\mathbb{R}}[0 ; 1]$, then $x \in L_{2}^{\mathbb{R}}[0 ; 1]$ and $R_{2}(x)=\|x\|_{L_{2}}=0$ if and only if $x=0$. We do not know does $R_{n}(x)=R_{n}(y)$ for every $n \in \mathbb{N}$ implies $[x]=[y]$ in the real case. 


\section{References}

[1] Ansemil J.M., Aron R.M., Ponte S. Behavior of entire functions on balls in a Banach space. Indag. Math. 2009,20 (4), 483-489. doi:10.1016/S0019-3577(09)80021-9

[2] Ansemil J.M., Aron R.M., Ponte S. Representation of spaces of entire functions on Banach spaces. Publ. Res. Inst. Math. Sci. 2009, 45 (2), 383-391. doi:10.2977/prims/1241553124

[3] Alencar R., Aron R., Galindo P., Zagorodnyuk A. Algebra of symmetric holomorphic functions on $\ell_{p}$. Bull. Lond. Math. Soc. 2003, 35 (1), 55-64. doi:10.1112/S0024609302001431

[4] Aron R.M., Falcó J., García D., Maestre M. Algebras of symmetric holomorphic functions of several complex variables. Rev. Mat. Complut. 2018, 31 (3), 651-672. doi:10.1007/s13163-018-0261-x

[5] Aron R., Falcó J., Maestre M. Separation theorems for group invariant polynomials. J. Geom. Anal. 2018, 28 (1), 393-404. doi: 10.1007/s12220-017-9825-0

[6] Aron R., Galíndo P., Pinasco D., Zalduendo I. Group-symmetric holomorphic functions on a Banach space. Bull. Lond. Math. Soc. 2016, 48 (5), 779-796. doi:10.1112/blms/bdw043

[7] Bornemann F. On the numerical evaluation of Fredholm determinants. Math. Comp. 2010, 79 (270), 871-915. doi:10.1090/S0025-5718-09-02280-7

[8] Chernega I., Holubchak O., Novosad Z., Zagorodnyuk A. Continuity and hypercyclicity of composition operators on algebras of symmetric analytic functions on Banach spaces. Eur. J. Math. 2020, 6, 153-163. doi:10.1007/s40879019-00390-z

[9] Chernega I., Galindo P., Zagorodnyuk A. Some algebras of symmetric analytic functions and their spectra. Proc. Edinb. Math. Soc. 2012, 55 (1), 125-142. doi:10.1017/S0013091509001655

[10] Chernega I., Galindo P., Zagorodnyuk A. The convolution operation on the spectra of algebras of symmetric analytic functions. J. Math. Anal. Appl. 2012, 395 (2), 569-577. doi:10.1016/j.jmaa.2012.04.087

[11] Chernega I., Zagorodnyuk A. Unbounded symmetric analytic functions on $\ell_{1}$. Math. Scand. 2018, 122 (1), 84-90. doi:10.7146/math.scand.a-102082

[12] Chernega I., Fushtei V., Zagorodnyuk A. Power operations and differentiations associated with supersymmetric polynomials on a Banach space. Carpathian Math. Publ. 2020, 12 (2), 360-367. doi:10.15330/cmp.12.2.360-367

[13] Einsiedler M., Ward T. Functional Analysis, Spectral Theory, and Applications. Graduate Texts in Mathematics, 276, Springer, Cham, 2017.

[14] Galindo P., Vasylyshyn T., Zagorodnyuk A. Symmetric and finitely symmetric polynomials on the spaces $\ell_{\infty}$ and $L_{\infty}[0,+\infty)$. Math. Nachr. 2018, 291 (11-12), 1712-1726. doi:10.1002/mana.201700314

[15] Galindo P., Vasylyshyn T., Zagorodnyuk A. The algebra of symmetric analytic functions on $L_{\infty}$. Proc. Roy. Soc. Edinburgh Sect. A 2017, 147 (4), 743-761. doi:10.1017/S0308210516000287

[16] Galindo P., Vasylyshyn T., Zagorodnyuk A. Analytic structure on the spectrum of the algebra of symmetric analytic functions on $L_{\infty}$. Rev. R. Acad. Cienc. Exactas Fís. Nat. Ser. A Math. 2020, 114, 56. doi:10.1007/s13398-02000791-W

[17] Gonzaléz M., Gonzalo R., Jaramillo J. Symmetric polynomials on rearrangement invariant function spaces. J. London Math. Soc. 1999, 59 (2), 681-697. doi:10.1112/S0024610799007164.

[18] Han J., Li Y., Lin L., Lu J., Zhang J., Zhang L. Universal approximation of symmetric and anti-symmetric functions. arXiv preprint, arXiv:1912.01765 [math.NA].

[19] Jawad F., Zagorodnyuk A. Supersymmetric polynomials on the space of absolutely convergent series. Symmetry 2019, 11 (9), 1111. doi:10.3390/sym11091111.

[20] Macdonald I.G. Symmetric Functions and Orthogonal Polynomials. University Lecture Series, 12, AMS, Providence, R.I., 1998.

[21] Martsinkiv M., Zagorodnyuk A. Approximations of symmetric functions on Banach spaces with symmetric bases. Symmetry 2021, 13 (12), 2318. https:/ / doi.org/10.3390/sym13122318 
[22] Mujica J. Complex Analysis in Banach Spaces. Elsevier, Amsterdam, 1986.

[23] Nemirovskii A.S., Semenov S.M. On polynomial approximation of functions on Hilbert space. Math. USSR Sb. 1973, 21 (2), 255-277. (translation of Math. Sb. 1973, 92(134) (2(10)), 257-281. (in Russian))

[24] Sunder V.S. Operators on Hilbert Space. Springer, Singapore, 2016.

[25] Vasylyshyn T.V. The algebra of symmetric polynomials on $\left(L_{\infty}\right)^{n}$. Mat. Stud. 2019, 52 (1), 71-85. doi: 10.30970/ms.52.1.71-85

[26] Vasylyshyn T. Algebras of symmetric analytic functions on Cartesian powers of Lebesgue integrable in a power $p \in[1,+\infty)$ functions. Carpathian Math. Publ. 2021, 13 (2), 340-351. doi:10.15330/cmp.13.2.340-351

[27] Zagorodnyuk A., Hihliuk A. Classes of entire analytic functions of unbounded type on Banach spaces. Axioms 2020, 9 (4), 133. doi:10.3390/axioms9040133

[28] Zagorodnyuk A., Hihliuk A. Entire analytic functions of unbounded type on Banach spaces and their lineability. Axioms 2021, 10 (3), 150. doi:10.3390/axioms10030150.

[29] Zaheer M., Kottur S., Ravanbakhsh S., Póczos B., Salakhutdinov R.R., Smola A.J. Deep sets. In: Luxburg U.V., Guyon I., Bengio S., Wallach H., Fergus R. (Eds.) Proc. of the 31st Intern. Conf. on Neural Information Processing Systems, Long Beach, California, USA, December 4-9, 2017. Curran Associates, Inc., Red Hook, NY, 2017, 3394-3404.

Received 14.02.2021

Revised 15.10.2021

Буртняк I., Чернега І., Гладкий В., Аабачук О., Новосад З. Застосування симетричних аналітииних функиій до спектрів лінійних операторів // Карпатські матем. публ. - 2021. — Т.13, №3. - С. 701-710.

Робота присвячена розширенню теорії симетричних аналітичних функцій на банахових просторах послідовностей на випадок просторів ядерних і $p$-ядерних операторів гільбертового простору. Введено алгебри симетричних поліномів і аналітичних функцій на просторах p-ядерних операторів, описано алгебраїчні базиси в таких алгебрах, знайдено зв' язки з визначником Фредгольма ядерного оператора. Крім того, розглянуто випадки компактних і обмежених нормальних операторів гільбертового простору і обговорено структуру симетричних поліномів на відповідних просторах.

Ключові слова і фрази: симетрична аналітична функція на банаховому просторі, $p$-ядерний оператор, визначник Фредгольма. 